\title{
eJRIEPS
}

Ejournal de la recherche sur l'intervention en éducation physique et sport

$41 \mid 2017$

Varia

\section{Enseigner la danse en EPS : réticences des enseignants et impact des formations initiales}

\section{Geneviève Cogérino}

\section{OpenEdition}

Journals

Édition électronique

URL : http://journals.openedition.org/ejrieps/687

DOI : $10.4000 /$ ejrieps. 687

ISSN : 2105-0821

Éditeur

ELLIADD

\section{Référence électronique}

Geneviève Cogérino, «Enseigner la danse en EPS : réticences des enseignants et impact des

formations initiales », eJRIEPS [En ligne], 41 | 2017, mis en ligne le 01 avril 2017, consulté le 01 août 2019. URL : http://journals.openedition.org/ejrieps/687 ; DOI : 10.4000/ejrieps.687

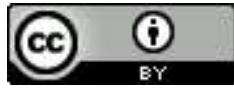

La revue eJRIEPS est mise à disposition selon les termes de la Creative Commons Attribution 4.0 International License. 


\section{Enseigner la danse en EPS : réticences des enseignants et impact des formations initiales}

Geneviève Cogérino, CRIS EA L-Vis, Université Lyon 1

\section{Résumé}

Le volume horaire consacré à l'enseignement de la danse et du cirque est relativement faible en EPS, même si ce dernier prend de l'importance. Des études antérieures ont souligné que l'habitus sportif des enseignants pourrait inciter au choix du cirque. L'article questionne les formations initiales (FI) et continuées (FPC) des enseignants d'EPS en danse. Le cadre psychosociologique postule le rôle central de l'articulation Savoir/Pouvoir de chaque enseignant sur le choix d'enseigner ou non ces pratiques. Quatorze entretiens semi-directifs ont été conduits auprès d'enseignants intégrant la danse et/ou le cirque, ou aucune des deux pratiques, dans la programmation EPS de leurs classes. Les questions portaient sur le déroulé et les ressentis à l'égard de leur FI et FPC en danse, leurs représentations des avantages ou inconvénients à enseigner danse ou cirque dans leurs classes. Les résultats précisent dans quelle mesure ces pratiques artistiques bousculent ou non l'habitus sportif de ces enseignants, créent des tensions entre réticences et obligations institutionnelles. Ils suggèrent les caractéristiques potentielles des Fl permettant à davantage de futurs enseignants d'introduire la danse en EPS : accroître " la confiance à enseigner la danse » plutôt que chercher à "faire connaître la danse ».

Mots clés : EPS, Formation initiale, danse, pratiques artistiques, psychosociologie

\section{Introduction}

Dans les programmes de l'EPS française, les pratiques physiques dites gymniques et artistiques rassemblent la danse, les arts du cirque, les gymnastiques rythmique et sportive, l'acrosport et l'aérobique. II s'agit d'y " concevoir, produire et maîtriser une prestation devant un public ou un jury, selon un code ou des règles de scène en osant se montrer et s'assumer ", selon la formulation des programmes du collège en 2008, par exemple (MENESR, 2008). Cet article se centre sur l'enseignement de la danse, et plus ponctuellement sur le cirque, dans le cadre de l'enseignement obligatoire avec pour seul intervenant l'enseignant d'EPS habituel de la classe. 


\section{eJRIEPS 41 avril 2017}

Le volume horaire effectivement consacré à la danse est faible, comparativement à celui octroyé aux autres activités possibles : $5 \%$ du volume horaire de l'EPS en $6^{\text {ème }}, 1 \%$ en classe terminale, de 1 à $2 \%$ en lycée professionnel, lors d'une étude sur 2500 enseignants d'EPS de six académies différentes (Terret, Cogérino \& Rogowski, 2006). L'enseignement du cirque tend à se substituer à celui de la danse en EPS. Associé aux activités physiques dites «artistiques», le cirque se rapproche simultanément des caractéristiques des pratiques sportives, avec la recherche de défi, de prouesses, de «performance » : altitude des sauts, prise de risque, acrobaties complexes. Les difficultés motrices ou techniques y sont aisément repérables (par exemple : jonglage à deux, trois ou quatre balles). Le cirque suscite également l'intérêt des élèves, même si celui-ci est variable selon les trajectoires scolaires des élèves et leur sexe (Garcia, 2007a ; Garcia \& Vigneron, 2006). II ouvre la porte, à l'insu des enseignants, à des braconnages, plus favorables aux garçons qu'aux filles (Garcia, 2007b), et proches de ceux constatés avec le hip hop, danse supposée « masculine » et propice à l'intérêt des garçons (Faure \& Garcia, 2003) : une hiérarchisation tacite et sexuée des rôles artistiques donne lieu par exemple à une monopolisation des monocycles par les garçons qui recherchent la prouesse, et la relégation des filles sur les ateliers plus chorégraphiques.

Les enseignants d'EPS globalement et les étudiants STAPS présentent un habitus sportif (Bourdieu, 1980) qui les éloigne des activités physiques artistiques (Garcia, 2007a ; 2013). Beaucoup ont une pratique physique personnelle compétitive et sont impliqués activement dans l'organisation du mouvement sportif en club (Benhaïm-Grosse, 2007). S'inscrivant dans une sociologie dispositionnaliste, Garcia (2013) démontre comment le cirque est un lieu propice au réinvestissement et transfert des ressources sportives antérieures des enseignants d'EPS, avec la morale de l'effort, le goût du dépassement de soi, l'attrait de la performance, le réinvestissement possible des prouesses gymniques et acrobatiques. Pour les enseignants d'EPS, et particulièrement les hommes, le choix du cirque souvent programmé par défaut permet de limiter l'engagement vers la danse, considérée territoire féminin et portée par des valeurs très différentes (Garcia, 2007a). II donne la possibilité d'affirmer que l'on enseigne en EPS une pratique physique artistique, conformément aux programmes, et l'enseignant peut y tenir à distance l'expressivité du corps, pourtant constituante d'une pratique physique de ce type. Cette expressivité s'investit davantage dans un jeu portant sur le personnage (dans le jeu d'acteur, celui du clown par exemple), sans se mettre au service de l'expression des émotions qui fonde les représentations de la 


\section{eJRIEPS 41 avril 2017}

danse scolaire. Les observations sociologiques soulignent que le cirque « gymnique » est largement présent en dépit de ses références artistiques. Mais cette approche permet aux enseignants hommes de sauver la face, en tant qu'hommes et sportifs, quand ils ont affaire à des élèves raillant les activités non-sportives : ceci laisse penser qu'ils font cirque «par obligation » (Garcia, 2007a).

Tenant compte du faible horaire consacré à la danse en EPS, on peut se demander si les formations initiales (FI) des futurs enseignants d'EPS sont pertinentes dans leurs objectifs et procédures pour donner la possibilité d'enseigner la danse à des étudiants majoritairement sportifs et peu connaisseurs des pratiques physiques artistiques.

1. 1. La formation n'est pas gage d'enseignement ultérieur Avoir "suivi » une formation en danse n'est pas le gage de son enseignement ultérieur. Au sein d'une recherche psychosociologique, Cogérino (1988) avait identifié quatre types de trajectoires formation/enseignement chez les enseignants d'EPS, à partir de 47 entretiens approfondis réalisés en 1983-84.

- Un tiers de ceux ayant bénéficié d'une FI intégrant la danse (8 sur 24) ne l'enseignaient pas à leurs élèves. Ces enseignants évoquaient majoritairement le « traumatisme » vécu lors des situations d'improvisation, épreuve qui était celle conçue pour la danse lors du recrutement par le CAPEPS. Le poids du regard des autres (impression d'être jugé, pesé, moqué...), le «manque d'idées », de savoir-faire, de « technique » pour ces improvisations dansées, l'impression pesante d'être « ridicule » étaient largement mentionnés et détaillés, lors de la description des vécus associés à la FI en danse. Les propos témoignaient d'une angoisse assez violente, d'une atteinte à l'estime de soi, liée à l'impossibilité d'incarner face à la classe le professeur « qui sait », « qui est capable ». En termes cliniques, c'était une atteinte à l'omnipotence qui était verbalisée, une mise en défaut simultanée du Savoir et du Pouvoir de l'enseignant (Cogérino, 1999) ;

- Deux tiers de ceux sans FI en danse (15 sur 23) proposaient des séances de danse et/ou d'activités d'expression à leurs classes. Leurs propos témoignaient d'une réflexion sur ce que pourrait être une « formation complète, diversifiée et équilibrée » des élèves en EPS. Cette proposition assumée se présentait comme une obligation morale pour ces enseignants. Celle-ci les avait conduits à rechercher des formations complémentaires, comblant les lacunes ressenties de leur FI. Ces formations recouvraient un panel d'activités souvent à la marge de l'EPS: théâtre, yoga, danse primitive, 
eutonie... La pression des programmes ou Instructions officielles n'était ni ressentie ni verbalisée explicitement ;

- Les huit sans FI en danse et qui ne l'enseignaient pas justifiaient leur choix par un fort sentiment de ridicule et d'inutilité des activités artistiques comparées aux pratiques sportives, incriminant une pratique sans Savoir: pas de contenus d'enseignement, pas d'apprentissages à viser, juste un « gigotage » sans intérêt. L'absence de FI était déclarée associée à une absence de « rencontre » avec un-e collègue plus chevronné-e qui aurait pu être observé-e, imité-e. Les angoisses de même nature que celles mentionnées dans le groupe des déçus de la Fl étaient repérables. Mais les interviewés de ce groupe projetaient leurs réticences et angoisses sur la nature même de l'activité, la danse ou les activités physiques d'expression, usant ainsi d'un mécanisme de projection pour maintenir la face et masquer simultanément leur « armure sportive ».

Au fil d'une recherche plus récente, Necker (2010) repère des traits communs chez les enseignants qui «osent» enseigner la danse: ils détiennent une culture artistique pratique (en suivant des cours pour eux-mêmes ou des stages qui ont assuré leur spécialisation en danse) ; leur sentiment de compétence naît de leur maîtrise d'une technique dansée ; ils appartiennent à des réseaux, des groupes informels qui donnent la possibilité d'échanger, dialoguer, construire et s'approprier une culture artistique dansée. Les enseignants novices en danse témoignent de craintes identiques à celles mentionnées dans les entretiens réalisés 25 ans plus tôt (Cogérino, 1988) : la peur du débordement et la perte $d u$ « contrôle » des élèves (le Pouvoir), la crainte du regard des élèves sur leur incompétence motrice en danse (le Savoir), le ressenti d'un péril associé à la manifestation involontaire d'émotions.

1. 2. Savoir faire ou avoir confiance en ses capacités ? Recherches récentes A l'heure actuelle, les programmes de l'EPS permettent de choisir des activités, ... et d'en éviter d'autres. Tel semble être le rapport entre danse et cirque. On peut se demander comment les enseignants d'EPS conçoivent et verbalisent le rapport entre danse et cirque. Ces représentations sont-elles en relation avec des procédures de leur FI, le vécu qu'ils en ont construit? Retrouve-t-on à l'heure actuelle les mêmes types d'itinéraires et d'argumentation, compte tenu des changements intervenus dans les FI et les formulations des programmes de l'EPS ? Des travaux réalisés entre ces deux périodes ont souligné chez les enseignants débutants l'impact stratégique de la confiance en leurs capacités à 


\section{eJRIEPS 41 avril 2017}

enseigner la danse pour l'introduire en EPS. Ces recherches ont aussi mis l'accent sur des procédures de formation plus efficaces que d'autres.

Un premier groupe de recherches a particulièrement suivi les enseignants polyvalents du premier degré qui doivent enseigner la danse, alors que leur formation y consacre peu de temps (Green, Chedzoy, Harris, et al., 1998 ; Hennessy, Rolfe \& Chedzoy, 2001 ; Rolfe, 2001).

Concernant le premier degré, ces recherches soulignent clairement l'impact de la mise en pratique, lors des stages dans les écoles, sur la confiance à enseigner la danse ultérieurement. La formation avant le stage peut être très brève (une dizaine d'heures) mais très efficace si elle est davantage centrée sur l'enseignement en classe: organisation de celle-ci, intervention à partir d'un type de danse -les danses collectiveschoisi pour accroître la confiance de l'étudiant en ses capacités à enseigner la danse. Le support octroyé par le tuteur de stage porte davantage sur l'encouragement que sur la technicité de l'intervention : ceci est perçu comme très efficace pour donner confiance aux étudiants. Ceux-ci ont vu leur anxiété diminuer, en dépit de leur faible niveau pratique personnel. La possibilité précocement introduite durant la FI d'enseigner la danse sous le regard encourageant des tuteurs de stage est donc un élément à retenir, même si l'impact des cours universitaires et de la pratique physique personnelle sont reconnus.

Un second groupe de recherches étudie plus spécifiquement le second degré (Connell, 2009 ; Lundvall \& Maivorsdottir, 2010 ; Mac Lean, 2007 ; Mc Cormack, 2001 ; Necker, 2010).

Pour ces étudiants, Mac Lean (2007) pointe des facteurs similaires, à la suite d'un suivi longitudinal sur quatre ans : l'expérience pratique en stage, au cours de la $\mathrm{FI}$, augmente leur confiance dans leur capacité à proposer et enseigner la danse en raison du soutien des tuteurs de stage. Le module universitaire est centré sur l'organisation des interventions (planifier, intervenir, évaluer) et constitue la plus forte contribution à la confiance. L'expérience physique personnelle antérieure en danse, plus élevée chez les étudiantes, n'occasionne pas de différences significatives sur l'habileté perçue à enseigner, comparée à celle des étudiants majoritairement moins expérimentés physiquement dans cette activité. L'ensemble de l'expérience en danse antérieure à la formation, des modules de formation universitaires et de la pratique en stage expliquent statistiquement $55 \%$ de la variance de la compétence perçue à enseigner la danse chez les étudiantes, $43 \%$ chez les étudiants. 


\section{eJRIEPS 41 avril 2017}

Mc Cormack (2001) a testé l'influence des pratiques réflexives sur l'attitude et l'habileté à enseigner la danse en EPS, à partir de la tenue d'un journal de bord, de questionnaires et de focus groupes. La formation associait cinq séquences de micro-enseignement en primaire durant un semestre, des cours universitaires (pratique physique -danse créative et danses collectives-, appréciation et évaluation de la danse et cours théoriques sur celleci). L'auteure constate de fréquentes appréhensions des étudiants à enseigner la danse en raison des souvenirs liés à leur pratique en tant qu'élève. Ceux-ci jouent un rôle de filtre pendant de nombreuses années sur la manière de percevoir et interpréter les contenus de leur formation. Elle souligne la nécessité d'examiner les valeurs et attitudes des étudiants à l'égard de la danse durant la formation, de développer l'habileté à créer, performer et évaluer la danse. La comparaison des réponses aux questionnaires de début et fin de session met en évidence une baisse des préjugés (la danse doit être enseignée par les femmes; des bases de danse classique sont nécessaires pour enseigner la danse; il faut enseigner des styles de danse convenant aux adolescents, etc.). L'accroissement de la confiance à enseigner la danse est manifeste.

Ces recherches s'incluent dans une diversité de systèmes éducatifs (Royaume-Uni, Australie, Suède). Mais les procédures de formation qui ont été constatées plus efficaces pour construire en FI la confiance des étudiants dans leur capacité à enseigner la danse en EPS sont similaires. II s'agit d'au moins deux aspects : 1. la précocité de la possibilité d'enseigner la danse (en stage, avec un tuteur) au cours de la FI, associée à des cours universitaires qui intègrent (et non se juxtaposent à) ces stages ; 2. l'absence d'allusions explicites à des connaissances qui renverraient à la différenciation des courants en danse contemporaine, à l'encontre ce que ce l'on peut constater dans les FI françaises.

Dès lors, on peut s'interroger sur ce que les enseignants d'EPS français ont gardé comme souvenirs de leur Fl et s'ils ont pu construire la confiance en eux pour enseigner la danse. Quel est l'impact de leur FI sur : -1 leur enseignement ou non de la danse, ou du cirque comme substitut ; -2 sur leurs capacités perçues à conduire des séances ?

\section{3. Cadre théorique}

Ces questions peuvent s'étudier sous l'angle des représentations sociales. Selon l'approche psychosociologique, les représentations sociales sont une association de préconceptions, images et valeurs, relatives à un domaine. Elles sont constituées autour d'une attitude (positive ou négative), colonne vertébrale d'autres manifestations psychiques comme les perceptions, les jugements ou comportements à l'égard d'un objet 
d'étude. Ces représentations forgent un système d'interprétation permettant de penser la réalité quotidienne: elles concernent la façon d'appréhender les évènements, les informations, les personnes et leurs relations. Elles contribuent à l'élaboration des comportements et des communications entre individus. L'ancrage des représentations se traduit par la signification et l'utilité donnée à l'objet étudié. Leur objectivation, construction sélective, renvoie à l'agencement et la forme des connaissances relatives à l'objet d'une représentation (Jodelet, 1984).

Lesne (1977) pose l'hypothèse que toute situation de formation (la situation d'enseignement en classe comme celle de formation professionnelle) peut être observée sous l'angle de deux rapports : le rapport au Savoir et le rapport au Pouvoir. Son approche d'origine sociologique établit que ces deux rapports constituent une structure dynamique sous-jacente à toute relation de formation. Le rapport au Savoir concerne les conceptions et opérations relatives aux contenus associés à une formation. Le rapport au Pouvoir concerne les conceptions et formes que prend la relation formateur-formé (formateur/étudiant ou enseignant/élève). Cette formalisation, issue de la formation d'adultes, résonne étroitement avec les propos de Pujade-Renaud (1976). Celle-ci étudie par questionnaires et entretiens les relations entre professeur et élèves lors de l'enseignement de la danse (36\% des répondants sont liés à l'EPS). Le rapport au Savoir est presque inévitablement un rapport au professeur car corps et savoir sont perçus «amalgamés » dans le contexte de l'enseignement de la danse. Elle pointe donc les ambivalences des rapports au Savoir et au Pouvoir et leurs étroites relations dans ce contexte, lors d'une démonstration par exemple.

Cet article vise donc à reconstruire les processus qui conduisent les enseignants d'EPS à enseigner ou non la danse, lui préférer parfois le cirque. Une démarche clinique compréhensive, une forme d'anamnèse des $\mathrm{Fl}$ est entreprise afin de cerner l'impact de leur déroulé sur les choix concernant ces pratiques physiques. L'approche de ces représentations repose sur l'hypothèse que les choix des enseignants sont tributaires de leur attitude à l'égard des pratiques artistiques, attitude construite sur l'articulation du Savoir et du Pouvoir. II est donc important de constituer des groupes de sujets à partir d'attitudes différentes à l'égard d'un même objet (ici l'enseignement de la danse en EPS). L'ancrage et l'objectivation des représentations de ces pratiques en EPS seront plus particulièrement étudiés au travers des questions suivantes : comment les enseignants d'EPS formulent-ils les avantages/inconvénients de la danse et du cirque ? Comment 


\section{eJRIEPS 41 avril 2017}

verbalisent-ils et/ou rationalisent-ils leurs choix ? Quel rapport au Savoir se dégage chez l'enseignant, concernant la danse comparée au cirque ? Le rapport au Pouvoir de l'enseignant à l'égard de la classe en est-il conforté ou bousculé ?

\section{Méthodes}

Le corpus analysé s'appuie sur des entretiens semi-directifs conduits auprès d'enseignants d'EPS dans les classes « ordinaires », sans dispositif d'enseignement spécifique. Quatorze entretiens ont été consacrés à la danse et au cirque. Les sujets (sept hommes, sept femmes) se différencient en fonction de leur âge (de 24 à 60 ans) et ancienneté professionnelle, établissement d'exercice (huit en collège, quatre en lycée et deux en lycée professionnel), volume horaire enseigné en danse ou cirque aux élèves, sentiment de compétence dans ces activités. Six exercent en zone ou réseau d'éducation prioritaire (ZEP/REP) ; ils ont été contactés en raison des fréquents arguments affirmant que l'enseignement des pratiques artistiques y serait plus difficile. Sur l'ensemble du panel, onze professeurs enseignent ou ont enseigné la danse, six le cirque, dont cinq ces deux activités, sur des volumes horaires variables.

Les entretiens visaient à obtenir :

- 1. des descriptifs des procédures d'apprentissage et de formation (en FI et FPC) et le type de danse support de leur FI ; éventuellement la nature de leur pratique personnelle (type de danse et quantité de pratique : régulière, occasionnelle...); la formulation des ressentis à l'égard de leur FI en danse; l'estimation de leur impact potentiel sur leur enseignement actuel ;

- 2. la formulation de leurs représentations de la danse et du cirque en EPS : « l'image » de ces activités, leur spécificité perçue, l'intérêt à les enseigner en EPS, l'énoncé d'arguments qui pourraient conduire à les intégrer ou exclure de l'EPS proposée à leurs élèves ;

- 3. les objectifs précis et procédures de guidage des élèves pour les enseignants d'EPS qui enseignent la danse, ou ce qu'il « faudrait faire » pour ceux qui ne l'enseignent pas (thématique qui ne sera pas développée dans ce texte : Cogérino, 2015).

Tous les enseignants interrogés ont suivi une formation concernant la danse durant leur $\mathrm{Fl}$, complétée pour certains par des formations volontaires ou des itinéraires de pratique physique personnelle (avant ou après leur $\mathrm{FI}$ ). Eu égard à la diversité des âges et périodes de recrutement au sein du panel, les FI suivies présentent des différences: 
certains ont eu une formation concernant le cirque et d'autres non. Les formations en danse ont été très diverses en durée, types de danse présentée (folklore, jazz, classique, modern dance, hip hop, contemporaine,...) et organisation (durée consacrée aux formations physique, didactique, culturelle ou pédagogique, par exemple). L'intensité de la spécialisation / expertise de chaque enseignant s'appuie sur deux éléments : 1. le type de $\mathrm{FI}$ : choix ou non d'une « option » de pratique en danse ou cirque ; participation à des ateliers chorégraphiques, etc. ; 2. la « quantité » d'enseignement de ces activités avec les élèves : nombre de séances conduites sur une année. Quatre niveaux de compétence en danse selon l'interviewé ont été caractérisés (aucune; faible; moyenne ; spécialiste) (Tableau 1).

Tableau 1. Principales caractéristiques des interviewés.

\begin{tabular}{|c|c|c|c|c|c|c|c|}
\hline $\begin{array}{l}\text { Pseudo } \\
\text { nyme }\end{array}$ & Sexe & Age & $\begin{array}{l}\text { Établis } \\
\text { sement }\end{array}$ & $\begin{array}{l}\text { Enseigne } \\
\text { actuellemen }\end{array}$ & & FI et FPC en danse & $\begin{array}{l}\text { Compétence } \\
\text { perçue }\end{array}$ \\
\hline & & & & Danse & Cirque & & Danse \\
\hline $\mathrm{H} 1^{*}$ & $\mathrm{H}$ & $20-30$ & $\mathrm{Clg}$ & non & non & FI STAPS** & aucune \\
\hline $\mathrm{H} 2$ & $\mathrm{H}$ & $30-40$ & LP & $\begin{array}{l}\text { non } \\
\text { Oui } \\
\text { avant***}\end{array}$ & non & $\begin{array}{l}10 \mathrm{~h} \text { en FI, didactique, } \\
\text { expression corporelle }\end{array}$ & aucune \\
\hline $\mathrm{F}$ & $\mathrm{F}$ & $30-40$ & Lyc & non & non & $\begin{array}{l}\text { FI Danse africaine ; } \\
3 \text { FPC }\end{array}$ & aucune \\
\hline $\mathrm{HC}+$ & $\mathrm{H}$ & $20-30$ & Clg ZEP & non & oui & FI STAPS 20h en L1 & moyenne \\
\hline $\mathrm{FC}+$ & $F$ & $30-40$ & Clg ZEP & $\begin{array}{l}\text { non } \\
\text { Rock acro*** }\end{array}$ & oui & $\begin{array}{l}\text { FI STAPS en D1 et } \\
\text { D2; } 1 \text { FPC }\end{array}$ & moyenne \\
\hline HD & $\mathrm{H}$ & $30-40$ & Clg & oui & non & $\begin{array}{l}\text { FI STAPS 20h en } \\
\text { contemporaine }\end{array}$ & faible \\
\hline FD+ & $\mathrm{F}$ & $50-60$ & Lyc ZEP & oui & non & Folklorique, jazz ${ }^{\star \star \star}$ & moyenne \\
\hline $\mathrm{HD}+$ & $\mathrm{H}$ & $30-40$ & Clg ZEP & oui & non & $\begin{array}{l}4 \times 20 \mathrm{~h} \text { en } \mathrm{FI} \\
\text { contemporaine } \\
1 \text { stage FPC }\end{array}$ & moyenne \\
\hline FD++ & $F$ & $50-60$ & Lyc & oui & non & $\begin{array}{l}\text { ExCo en FI ; } \\
\text { contemporaine en } \\
\text { FPC puis en } \\
\text { Association }\end{array}$ & spécialiste \\
\hline $\mathrm{HD}++$ & $\mathrm{H}$ & $30-40$ & LP & oui & non & $\begin{array}{l}\text { Danseur Hip hop dans } \\
\text { une troupe ; Ateliers } \\
\text { chorégraphiques; } \\
\text { Anime stages FPC } \\
\end{array}$ & spécialiste \\
\hline $\mathrm{F} 1 \mathrm{CD}++$ & $F$ & $30-40$ & Clg & oui & oui & $\begin{array}{l}\text { FI Option danse } \\
\text { Contemporaine; } \\
\text { modern jazz, hip hop }\end{array}$ & spécialiste \\
\hline FCD+ & $\mathrm{F}$ & $30-40$ & $\mathrm{Clg}$ & oui & oui & 3 cycles en $\mathrm{FI}$ & moyenne \\
\hline $\mathrm{HCD}+$ & $\mathrm{H}$ & $30-40$ & Clg ZEP & oui & oui & $\begin{array}{l}\text { Ateliers } \\
\text { chorégraphiques en } \\
\text { FI, } 2 \text { stages FPC }\end{array}$ & moyenne \\
\hline $\mathrm{F} 2 \mathrm{CD}++$ & $\mathrm{F}$ & $50-60$ & Lyc ZEP & oui & oui & $\begin{array}{l}\text { Contemporaine, danse } \\
\text { acro, danses de salon, } \\
\text { FI + FPC }\end{array}$ & spécialiste \\
\hline
\end{tabular}




\section{eJRIEPS 41 avril 2017}

$\left(^{*}\right)$ La première lettre indique le sexe, suivie du chiffre 1 ou 2 si deux sujets ont les mêmes caractéristiques professionnelles ; C et/ou D pour « enseigne Cirque et/ou Danse » ; le niveau de compétence perçue est signalé par : $(+)$ : moyenne ; (++) spécialiste.

$\left({ }^{* *}\right)$ L'interviewé donne peu d'informations précises sur cette formation.

$\left.{ }^{* * *}\right)$ L'interviewé a eu l'occasion d'enseigner la danse dans un établissement précédent.

Une analyse de contenu thématique, de type inductif, a été acheminée. Tous les entretiens ont été transcrits intégralement (corpus de 242700 caractères, questions et relances exclues), découpés en items sur la base thématique, puis regroupés a posteriori et progressivement en catégories plus larges (annexe 1). Par ailleurs, 82 termes (détaillés en 3.4) ont été repérés à l'aide de la fonction Rechercher et comptabilisés au sein de chaque entretien.

\section{Résultats}

Ces résultats seront présentés dans l'ordre suivant: la $1^{\text {ère }}$ partie consacrée à la $\mathrm{FI}$ abordera celle-ci sous l'angle des souvenirs marquants qu'elle a laissés aux étudiants, des reproches formulés par les enseignants à son égard, puis de l'impact de la présentation des divers types de danse en FI. La $2^{\text {ième }}$ partie évoquera la FPC qui, par contraste, présente des caractéristiques positives aux yeux de ces enseignants. La $3^{\text {ième }}$ partie fera le point sur la balance entre Danse et Cirque : les doutes, avantages et inconvénients spécifiques, perçus par les enseignants. La $4^{\text {ième }}$ partie, plus brève, pointera quelques spécificités du lexique utilisé par les interviewés, selon qu'ils enseignent Danse, Cirque ou ni l'une ni l'autre.

3. 1. La formation initiale

Les souvenirs et ressentis mentionnés par les interviewés sont une élaboration mentale, mémorielle, cognitive et affective, propre à chacun. Les souvenirs ne sont pas une description du réel et le « vécu » s'éloigne de ce fait d'un strict « récit » du décours de la FI suivie. Néanmoins, accéder à ces souvenirs de FI permet de comprendre les refus ultérieurs des enseignants à se «lancer » dans l'animation d'un cycle de danse avec leurs élèves, et leur préférence à proposer le cirque aux élèves.

\section{1. 1. Les souvenirs et ressentis issus de la FI}

Tous les interviewés ont participé en FI à une formation en danse (même s'ils déplorent sa brièveté). De manière peut-être symptomatique, ce sont d'abord des souvenirs 


\section{eJRIEPS 41 avril 2017}

émotionnels qui sont mentionnés, bien davantage que le repérage des apports techniques ou didactiques: «rigolades » en groupe en prenant conscience de son incoordination, sentiment de découverte ou profond ennui face à un univers inconnu, « choc » en découvrant les procédures d'échauffement corporel telles que les frictions, embarras face à l'obligation de montrer aux autres sa prestation ou fierté d'avoir survécu à cette épreuve. «Une sensation bizarre parce qu'on avait peur de tout ça, alors un peu la peur du regard des autres, et en même temps ça provoquait des fous rires parce que, on était un petit groupe, on n'avait aucune souplesse [...]. C'était super agréable de pouvoir se défouler à travers la danse, et se rendre compte que c'était quand même assez physique, qu'on était bien crevé quand même parfois en sortant » $(\mathrm{H} 1)$.

L'impression laissée par un premier contact avec la pratique de la danse, inconnue antérieurement, n'est pas systématiquement négative. Ces deux avis contrastés sont formulés par des enseignants qui n'ont pas introduit la danse dans leur enseignement : « C'était une horreur, très mal vécu [...] il fallait être un peu disjoncté pour danser. 》 $(\mathrm{FC}+)$; « Je n'en garde pas une impression négative bien au contraire, c'est un souvenir positif, j'ai découvert une activité que je ne connaissais pas. » $(\mathrm{HC}+)$ Des souvenirs un peu «tendus" sont aussi mentionnés par ceux qui enseignent à l'heure actuelle la danse : « Je me suis mise à la contemporaine avec un petit peu de souci au début, surtout pour me montrer, comme tout le monde. 》 (F2CD++).

Ces ressentis négatifs n'ont pas été un obstacle insurmontable ultérieurement : certains ont dépassé cette phase et enseignent actuellement la danse à leurs élèves, tout en déclarant qu'ils ont même « oublié » en quoi consistait leur FI. D'autres apprécient d'avoir été confrontés à une forme d'épreuve qui leur permet aujourd'hui de mieux comprendre certains comportements d'élèves face à l'activité : « Le fait de passer avec des choses à exprimer justement autour d'un thème et toute une gestuelle à inventer, je trouve ça nous implique plus, nous en tant qu'individu, lorsqu'on présente et euh... La peur de passer devant les gens est décuplée. Euh... ouais justement c'est intéressant ça, de passer devant les autres en se déballant un peu, donc j'en garde un bon souvenir. [...]. J'enseigne des tas de trucs que j'ai jamais vécus, alors je... ouais, je pense qu'un vécu en danse c'est bien! 》 (HD)

II arrive que des moments purement anecdotiques soient « le » souvenir positif qui émerge de la FI : «Ça a été une évaluation que j'ai faite où je prenais plaisir, j'étais fière de ce que l'on avait fait avec mon groupe, quoi. Contente de cette évaluation-là. J'ai toujours cette 


\section{eJRIEPS 41 avril 2017}

image en tête à chaque fois. On a fini avec un porté, et on était trois filles et on portait le garçon en fait. » (FCD+) Pour d'autres les souvenirs positifs renvoient à la différence perçue entre les pratiques sportives « habituelles» et la danse : «Super souvenir... [...] Tu connais les gens différemment, tu n'es pas sur le pôle compétition et ça nous faisait du bien aussi à nous...Ce qui est reposant en danse, c'est qu'il n'y a pas de tricherie, du genre:"tu m’as battu, je pose réclamation"... Et ça, c'est le bonheur... On y va parce qu'on a envie de le faire, après c'est sûr que de temps en temps on a envie de gagner mais ça permet de relativiser...! » (F2CD++)

II arrive aussi que le primat de souvenirs émotionnels négatifs soit lié à l'inadéquation des procédures didactiques de la $\mathrm{Fl}$. C'est ce qu'évoque cette interviewée, sommée de s'exprimer en danse africaine au son d'un djembé : «On jouait sur du djembé, on devait s'exprimer, enfin c'était franchement très particulier et, pour moi, je ne me vois pas enseigner cette forme de danse ! [...] La danse africaine, un grand moment !!! kong-kakong-ki-ki-kong-ka-kong! Voilà, il fallait danser là-dessus, sur des rythmes intériorisés, et en bougeant le plexus d'avant en arrière. Donc, vous imaginez ce que ça peut donner pour des gens en plus pas forcément... Enfin, moi personnellement, j'avais jamais vécu de cycle danse en tant qu'élève avant. Alors j'arrive, future prof d'EPS, ben, on me dit la danse c'était ça [inspiration]... Là, tout de suite, on n'a pas envie d'enseigner la danse ensuite. » (F) Une autre interviewée détaille le récit d'une expérience malheureuse selon elle : «Souvenir négatif : ... on était par petits groupes, on devait être 5/6, il fallait que l'on se regarde alors, il fallait faire un certain rythme avec les pieds et tout. Et à un moment donné, il fallait faire [mouvement, grande respiration : démonstration] se lâcher et puis se vautrer par terre et tout, et euh... voilà, cette situation-là. Et elle trouvait qu'on ne se relâchait pas bien, qu'on était trop nana... et puis que on se relâchait trop vite, on faisait trop de bruit en tombant... Et puis on a répété cette situation-là « $x$ » fois et je me souviens qu'il fallait [grande respiration, démonstration gestuelle bras], se lâcher par terre et tout... et voilà, en plus on se cognait les uns... Enfin, cette situation-là, elle m'a marquée et voilà, et elle insistait sur : on ne se relâchait pas bien, on ne se jetait pas bien par terre... Et voilà après ça a dégénéré totalement... Après on a commencé à faire des plongeons de piscine et tout ça [rire]. Voilà, ça m'a marquée parce que cette pauvre prof elle a perdu prise à un moment donné sur notre groupe [...]. Et puis on a répété ça « $x$ » fois et, ouais, j'avais l'impression que c'était un mélange de sophrologie, de yoga en échauffement et tout... Enfin je ne comprenais pas l'intérêt, et je ne comprenais pas après 


\section{eJRIEPS 41 avril 2017}

la relation après avec le travail que l'on faisait et ... » (FC+). Ainsi, l'intense sentiment d'incompréhension, la lassitude associée à cette vaine répétition conduisent dans la suite de l'entretien à la formulation d'un rejet de la danse. Pour d'autres, les a priori négatifs liés à la danse contemporaine sont progressivement atténués par la découverte d'un rapport au corps, de l'écoute de soi chez ceux qui enseignent la danse actuellement : «...plus la connaissance de soi, la maîtrise de soi sur ce qui est kinesthésique, proprioceptif, cette dissociation segmentaire. Donc d'une part technique, et de l'autre c'est la liberté. La liberté d'expression [...] On n'est pas fermé contrairement à d'autres danses ou pratiques où il faut respecter des règles. En danse, on a une liberté d'expression, je ne dirais pas sans limites mais quasi. N'importe qui peut réussir à exprimer quelque chose. |...] C'est cette liberté de pouvoir avoir une pratique sans être enfermé dans une obligation, dans une règle du jeu kinesthésique, proprioceptif. 》(F1CD++)

Plusieurs entretiens mentionnent explicitement leur refus d'enseigner la danse, face à ce qui leur apparaît « incompréhensible », insupportable dans la danse... Les souvenirs négatifs concernent selon les cas la danse elle-même et le rejet qu'elle suscite, ou les modalités de son enseignement en FI. II est à noter que les apports techniques et didactiques des $\mathrm{FI}$ sont très peu mentionnés dans les entretiens. Le silence les concernant est-il vraiment dû à leur absence ou aux procédures des FI qui ne les ont pas mis en valeur pour les étudiants ?

\section{1. 2. Les reproches formulés à l'encontre des FI}

On peut résumer les reproches formulés à l'encontre de la $\mathrm{FI}$ en danse aux points suivants : un choc à la découverte d'une pratique physique très éloignée des enjeux plus habituels des pratiques sportives (gagner, se dépasser...) ; le constat de son inculture chorégraphique et donc l'absence de repères pour resituer la diversité des courants de danse ; un vécu négatif lié à la prise de conscience de son niveau de « débutant », ressenti assez inhabituel de la part d'étudiants engagés en STAPS; l'absence de présentation de « vrais élèves » durant la $\mathrm{Fl}$ et de repères didactiques et pédagogiques qui permettraient de gérer les séances face aux élèves : «Moi à la formation que j'avais eue, voilà, ils avaient montré des vidéos [...], pas d'élèves en fin de compte, [mais] de danseurs quoi... on était parti de ça. » (FC+) En l'absence d'élèves, les situations d'apprentissage présentées en FI sont déclarées « peu exploitables ».

Pour les enseignants qui n'enseignent pas la danse, les tensions sont manifestes entre la présentation d'outils didactiques et pédagogiques, et/ou la construction d'une culture 


\section{eJRIEPS 41 avril 2017}

permettant de se repérer au sein des divers courants de danse : «On a pratiqué, après il y avait une petite formation théorique mais sincèrement c'était plutôt lié à la connaissance de l'activité. [...] Mais je me souviens qu'on nous avait montré des vidéos des spectacles de Découflé, de... d'autres chorégraphies quoi, pour nous montrer différents styles de danse. Mais sincèrement, pour notre culture personnelle c'était intéressant, mais en terme d'exploitation pédagogique et didactique...chaud, vraiment chaud. [...] J'ai pratiqué la danse mais dans des conditions tellement éloignées de ce que l'on peut connaître en milieu scolaire que c'est peu ré-exploitable quoi ! » ( $\mathrm{HC}+)$

L'association d'un vécu « pratique » ou « technique » négatif durant la FI avec l'absence de perception du sens de l'activité est une source majeure du refus d'enseigner la danse ultérieurement : "La danse, à quoi ça sert ? ». " J'ai fait un deuxième cycle de danse, type..., je ne sais pas comment on appelle ça: de l'expression corporelle, danse contemporaine? Enfin voilà...où on a, chaque groupe, c'était un peu affinitaire, et on retrouvait vraiment les garçons ensemble, très typés. [Je ne] sais pas comment expliquer... dans du « copiage » d'autres activités physiques classiques : tennis de table, $d u$ sport co... On essayait de, transformer à travers la danse... et puis jusqu'[aux] filles qui étaient spécialistes danse et qui présentaient des spectacles vraiment poussés. 》 $(\mathrm{F})$ Les efforts des formateurs/trices pour suggérer des pistes faciles pour l'enseignement ne sont perçus que sous l'angle de leur décalage avec les représentations des étudiants (le « copiage d'APS»).

II en ressort que parfois les souvenirs négatifs vécus en FI en danse l'emportent sur les souvenirs positifs. Les apports de repères pour l'intervention, la conception et la régulation des séances (s'ils ont été présentés) semblent avoir été oubliés ou rejetés des mémoires. Les enseignants gardent en mémoire davantage d'inconvénients à pratiquer la danse, comparativement aux bénéfices qu'en tireraient les élèves. Ils déclarent avoir été informés de divers courants de danse contemporaine mais ne pas savoir que faire de ces connaissances. Ce déficit du Savoir entame leur confiance à enseigner la danse.

3. 1. 3. Les types de danse proposés en FI

Concernant les types de danse (folklore, danse jazz, africaine, contemporaine....), ceux qui ont été présentés sont divers, en fonction des périodes où ont été formés les étudiants. Dans le corpus des entretiens, les types de pratiques physiques artistiques mentionnés sont : cirque (76 fois) ; contemporaine (29) ; hip hop (23) ; modern jazz (8) ; rock (7) ; 


\section{eJRIEPS 41 avril 2017}

mime (6) ; danse classique (6) ; théâtre (5) ; boîte de nuit (3) ; jazz (3) ; danses collectives (2) ; salsa (2). Le nom seul « danse » est énoncé 398 fois, le verbe « danse » : 18 fois.

Pour les plus récentes formations, quelques sous-courants de danse contemporaine dominent. C'est particulièrement le cas de la danse-théâtre : Pina Bausch est la plus souvent citée. Ce courant occasionne un enthousiasme de la part des «spécialistes ». Chez les moins convaincus de l'intérêt d'enseigner la danse en EPS, il conduit à l'énoncé d'un rejet ou d'un malaise.

En tant que pratique sociale, incluse dans la culture, donnant lieu à des spectacles, la danse (surtout contemporaine) semble véhiculer une image négative aux yeux de la plupart des enseignants d'EPS et des étudiants en STAPS: " J'avais un peu la représentation de la danse contemporaine le truc mort, assez froid, on [ne]comprend rien, très difficile à rentrer dans leur univers, quoi. » (HD+) ; “ Je partais avec tous les a priori de la danse contemporaine où c'est un truc un peu bizarre, on se demande si c'est vraiment de la danse. » (HD++) ; «Moi, la danse contemporaine, je suis un peu critique parce que je la trouve trop..., elle est devenue à mon goût trop intellectualisée [...], quand on [n'] y est pas préparé, quand on [n'] est pas dans le monde de la danse contemporaine, je dirais "pure" pour simplifier. Voir un homme qui arrive nu sur scène, qui arrive et qui repart, pour moi ça n'exprime pas grand-chose. Mais voilà, ce n'est que mon avis personnel. » $(\mathrm{F} 1 \mathrm{CD}++)$ Ces trois interviewés enseignent actuellement la danse à leurs élèves. On peut donc supposer que d'autres éléments ont contribué ultérieurement à modifier cet a priori négatif. Mais d'autres enseignants n'ont pas été en mesure de réélaborer cette image négative pour pouvoir oser enseigner la danse à leurs élèves. La FPC est une occasion potentielle de cette réévaluation de l'intérêt de la danse, encore faut-il avoir envie de s'y inscrire...

\section{2. Une FPC plus opérationnelle?}

La qualité de la FI joue un rôle important, mais sa brièveté ne permet pas toujours de dépasser les réticences des futurs enseignants, ni de les aider à comprendre ultérieurement leurs difficultés à organiser, gérer les modalités concrètes d'enseignement. En revanche, les sessions de FPC sont vues de manière beaucoup plus positive. Les arguments sont d'un grand classicisme. L'animation par de « vrais profs » qui ont de « vrais élèves » emporte l'adhésion. L'enseignante, qui relatait son désarroi face à l'injonction de « s'exprimer » au son du djembé, mentionne plus tard: «Donc avec des gens qui, eux, étaient plus sûrs ; enfin, c'était des vrais profs d’EPS, ceux qui ont enseigné 


\section{eJRIEPS 41 avril 2017}

avec leurs élèves et qui faisaient voir différents types d'approches avec leurs élèves, voilà! Et c'est plus à travers ces FPC professionnelles que j'ai appris, que j'oserais me lancer maintenant dans l'activité danse avec mes élèves si elle était programmée, pas que ce que j'ai vécu en formation initiale ! On en retire déjà des fiches, des situations motrices utilisables avec nos élèves directement. Donc après il faut effectivement se lancer et les tenter pour voir celles qui correspondent peut-être plus ou moins à son public. » $(F)$

La FPC est perçue «efficace » si elle parvient simultanément à donner confiance à l'enseignant (en dépit de souvenirs ou expériences négatives liées à la FI, et même s'il n'a eu aucune pratique physique antérieure dans ce domaine) et à donner des repères sur l'activité des élèves confrontés à la danse. Les sessions où on « voit de vrais élèves », où on vit les mêmes situations qu'eux, les mêmes "formes de pratiques scolaires » sont donc particulièrement soulignées et plébiscitées. Les enseignants opposent clairement les insuffisances d'une FI uniquement «culturelle », se limitant à présenter l'histoire et la diversité des œuvres scéniques, ou «théorique»: «Prétendre en théorie avoir suffisamment de savoir pour pouvoir enseigner l'activité... Enfin, moi ça ne me paraît pas possible, sinon ça serait simple... Il suffirait d'ouvrir un bouquin, quel qu'il soit, et on serait capable de l'enseigner... Ça ne me paraît pas possible comme ça ! Et sinon je pense qu'il y aurait beaucoup de collègues dans les établissements qui se seraient lancés si ce n'était qu'une question de connaissances théoriques... Vraiment, ouais, il faut avoir pratiqué ! » $(\mathrm{HC}+)$

Les enseignants déjà chevronnés apprécient les échanges entre collègues qui les « ressourcent » : «Entre collègues on discute et on voit, on échange, on partage... On évoque surtout l'entrée dans la danse, comment lancer le cycle pour motiver les élèves donc ça on se donne... Ça nous permet de nous voir entre profs pratiquant l'activité... Ce qui est intéressant c'est de parler entre nous, puisqu'on a nous aussi des niveaux de pratique différents... Et puis aussi pour tout ce qui est exercices... parce que, finalement, on vient prendre des recettes. 》 (F2CD++) II s'agit aussi de sortir de ses routines, mieux comprendre les nouveaux programmes: «Ça m'a rafraîchi des idées sur des petites situations, ça m'a également permis de mieux comprendre les attentes au niveau du programme et de mieux aussi estimer ce que l'on pouvait attendre d'un élève... après un cycle, après un deuxième cycle, etc. Parce que j'avais un peu tendance à faire la même chose, que ce soit un premier cycle, un deuxième cycle... à attendre un peu les mêmes 


\section{eJRIEPS 41 avril 2017}

choses. Je n'avais pas de vision de progression. [...]. Alors ça m’a vraiment clarifié les choses, enrichi au niveau des idées. 》 (FCD+)

En synthèse les sessions de FPC sont plébiscitées car elles prennent la formation par l'entrée professionnelle : comment s'y prendre avec les élèves pour les faire adhérer, leur permettre de progresser, comment éviter la routine ou en sortir, comment être dans le respect des programmes, comment comprendre, analyser, évaluer les comportements des élèves... ? La pratique physique qui y est sollicitée est celle qui correspond à la réalisation des situations d'apprentissage ou des formes de pratiques telles que celles proposées aux élèves. L'enseignant se réapproprie le Pouvoir auprès de sa classe par la confiance en un Savoir reconquis. Aucune mention de craintes ou de ressentis négatifs liés à la pratique de la danse n'émerge des entretiens lorsque qu'on aborde l'impact des FPC.

\section{3. Entre réticences et obligations institutionnelles}

\section{3. 1 Subjectivité et démonstration : Charybde et Scylla ?}

Les obstacles perçus à l'enseignement de la danse se livrent en sous-couches successives: les arguments se différencient progressivement au fil de l'entretien pour atteindre ceux impliquant davantage l'enseignant.

Quelques interviewés s'avèrent réticents d'emblée à programmer la danse avec leurs élèves, invoquant de multiples raisons : installations matérielles inadéquates ; réactions et réticences des élèves; connotation genrée de la danse; incompétence perçue de l'enseignant; équipe professorale majoritairement ou exclusivement masculine ; inadéquation de la $\mathrm{FI}$; inintérêt de la danse elle-même ; préférence pour une activité plus dynamique et conforme à l'habitus sportif: «Je suis plus sur l'activité physique de dépassement de soi, transpiration, effort physique où on sent l'effet sur son corps [...] une danse physique, le rock'n'roll par exemple ! » (H2)

Les élèves sont resitués comme manifestement en refus vis-à-vis de cette activité : les représentations de la danse chez les élèves («danse avec les stars », «le show », ou « boîte de nuit, tutu, ballerine, activité de fille» (HCD+). La peur de se toucher et la pudeur de certains, la peur du jugement des autres, leur manque de maturité sont évoqués: «Public jeune,... pas encore sur.., ils n'en sont pas au dialogue sur l'art, du tout ! Ils sont dans le ressenti. » (HCD+)

L'intérêt « éducatif » de la danse est ainsi remis en cause : « Je me dis que ça peut être violent pour des gamins [...] On a l'impression qu'il y a moins d'ouverture, qu'il y a moins de choses auxquelles se raccrocher, les élèves vont moins s'y retrouver. " (FC+) $\mathrm{Ce}$ 


\section{eJRIEPS 41 avril 2017}

propos résonne comme une projection sur l'élève de préoccupations, de craintes propres à l'enseignant lui-même. Car la compétence de l'enseignant, le rapport au Savoir, apparaît vite au centre du problème : «On n'a pas d'objets sur lesquels se raccrocher, je pense que ça nous fait plus peur d'enseigner la danse, parce qu'il n'y a pas ces supports-là ! [...] Le manque de pratique et de connaissance de l'activité nous a fait nous tourner vers le cirque. La danse, ça me paraît moins concret pour moi, en tant qu'enseignant, de matérialiser le progrès des élèves. 》 $(\mathrm{HC}+)$

Deux thèmes principaux constituent l'armature des doutes énoncés à l'égard de la danse : sa «subjectivité » et la crainte d'une démonstration à effectuer. La thématique de la subjectivité est attribuée à la danse de manière "constitutive » et à l'évaluation de la chorégraphie, le plus souvent notée à la fin du cycle. L'ensemble constitue un frein notable à l'enseignement de la danse pour ceux qui ne l'enseignent pas, parmi lesquels ceux qui enseignent seulement le cirque (tableau 2). Le fait que la danse contemporaine puisse partir de n'importe quelle thématique, action motrice ou contrainte de composition déstabilise les enseignants : la diversité et la multiplicité des possibles les submergent et leur donnent l'impression que l'on peut faire «tout et n'importe quoi », sans point de repères clairs. Par ailleurs, les spectateurs ne perçoivent pas les mêmes attraits, ou les mêmes qualités, ou le même «thème » dans une chorégraphie. Ces enseignants la considèrent donc comme (trop) subjective : « [cette subjectivité] elle est indéniable parce que, par rapport à l'émotion que la danse peut créer... c'est la partie subjective d'une personnalité par rapport à l'organisation, les effets qui peuvent être mis en scène. Telle ou telle personne n'aura pas la même réaction par rapport à d'autre, quoi ! Donc, la subjectivité elle est là, quoi ! L'émotion créée, ce qui est compris, ressenti de la danse ! » (H2) La méfiance ou le rejet de la subjectivité inhérente, dans leurs propos, à la danse contemporaine se présente comme une mise à distance de l'émotion, source potentielle d'inspiration ou de composition, mais aussi du ressenti des spectateurs : « Le problème est qu'il y a toujours une notion de subjectivité dans la danse. [...] A part une activité, purement centrée sur l'aspect technique, euh... quand on parle de danse, il y a toujours une part de subjectif, d'émotions que cela suscite chez le spectateur etc. » (H1) Ce que ce groupe d'enseignants englobe aussi sous le terme " subjectivité » renvoie d'une part à la thématique de l'émotion ; d'autre part, à ce qui est présenté en formation comme les «variantes », les «paramètres », la «trame de variance » sur lesquels le chorégraphe peut jouer pour modifier, nuancer, améliorer une prestation dansée. 


\section{eJRIEPS 41 avril 2017}

La subjectivité est aussi celle de l'enseignant évaluateur/notateur d'une prestation artistique. Le cycle consacré à la danse ou au cirque se conclut toujours par l'attribution d'une note pour l'élève. Cette notation est partiellement assumée par ceux qui enseignent la danse, s'en remettant à force tableaux et grilles d'évaluation critériée. Ils évitent ainsi que les élèves se sentent notés aléatoirement : "La difficulté c'est d'arriver à noter les élèves pour le bac, la part de subjectivité soit toujours présente. [...] On essaie de la réduire le plus possible avec des tableaux... [...] Le plus subjectif c'est la choré[graphie], on note aussi la technique : amplitude, vitesse, énergie. » (F2CD++) Pour les enseignants moins attirés par la danse, la notation est vécue comme l'exemple type de la subjectivité du spectateur (le professeur), en rupture avec les habitudes classiques de notation, largement étayées sur des barèmes affichés et connus des élèves. La notation sommative (et certificative en classe terminale) est donc une « épreuve » vécue par l'enseignant, se sentant pris en flagrant délit de notation aléatoire : « Et on va dire, tout ce qui est ressenti émotionnel, ça doit garder une part infime et... comme justement c'est non-quantifiable, c'est pas forcement compréhensible par les élèves... " Pourquoi à machin vous lui avez mis tant ?... alors qu'à moi... ". Donc cette part-là, la part émotion quelque part, elle doit être limitée quoi! La part de subjectivité... » (F) Pour contourner l'obstacle de cette subjectivité, certains envisagent des repérages et comptages d'actions motrices (y a-t-il bien dans la chorégraphie présentée tant de tours, de sauts, de passages au sol ? L'espace est-il occupé entièrement ?). Ainsi, une mélodie conçue avec « toutes les notes de la gamme », un tableau utilisant «toutes les couleurs » seraient-ils des œuvres artistiques plus achevées... L'aboutissement de ce type d'approche conduit donc la nonprogrammation de la danse, ceci permettant de ne mettre en difficulté ni les élèves ni l'enseignant.

Des allusions indirectes laissent percevoir que l'enseignant n'est pas en difficulté seulement sur le terrain de ses compétences professionnelles, au plan du savoir et de la gestion de la classe : «Une activité particulière dans la mesure c'est assez difficile de se montrer au regard des élèves et de gérer cette activité. [...] La réticence, c'est une activité où il faut oser. Pour que les élèves démarrent, il faut que le prof montre, contrairement à d'autres activités. En sport collectif ou quoi, on n'est pas obligé de... montrer l'activité pour que les élèves entrent facilement dans l'activité. [...] Je pense qu'en danse, il faut vraiment se lancer, se lâcher. » (F) La crainte de ne pouvoir démontrer si nécessaire est énoncée par ceux qui n'enseignent pas la danse. La compétence professionnelle de l'enseignant 


\section{eJRIEPS 41 avril 2017}

est intuitivement perçue comme défaillante et bousculée, lors des commentaires relatifs à l'utilité, la justification de l'usage de la démonstration en danse (Cogérino, 2015). L'atteinte narcissique est difficilement acceptable : les enseignants se perçoivent non-omniscients, incompétents et «jugés par les élèves ». Ils préfèrent alors renoncer à la danse plutôt qu'oser la « démaîtrise ».

\section{3. 2. L'apport/intérêt de la danse pour les élèves}

Sur cette thématique, les perspectives énoncées se différencient selon que les interviewés enseignent ou non la danse à leurs élèves.

- Chez ceux qui ne l'enseignent pas, l'intérêt concerne : l'apport de « davantage de créativité » ; un rapport au corps plus inhabituel ; un « enrichissement des gestuelles » ; le développement d'une conscience corporelle ou de "dimensions du corps qu'ils ne connaissent pas »; la sollicitation d'élèves plus en retrait dans les autres activités. Si elle a été abordée en $\mathrm{FI}$, la clarification autour de la dimension spécifiquement artistique a laissé peu de traces et souvenirs précis : sa mention n'apparaît qu'une seule fois dans le corpus sous la forme: “développer l'émotivité de l'élève, développer sa sensation artistique». Les formulations sont souvent générales et généreuses. Elles listent des apports que d'autres activités ou d'autres organisations de séances pourraient apporter: donner la « notion de projet », «le savoir communiquer dans le groupe, l'entraide dans le groupe, la communication, la création dans le groupe. Est-ce qu'on est capable de s'entendre, de créer ensemble ?! Ce n'est pas un qui crée et les autres qui..., enfin, un qui a les idées et les autres qui suivent... Il faut l'émanation de tout le groupe... Pour moi, c'est ça le versant éducatif, plus la communication, la création dans le groupe, le savoirêtre, savoir communiquer ensemble ! » (F)

La «prestation artistique » est envisagée davantage comme «épreuve » et non comme une échéance, un aboutissement, parfois temporaire : “C'est aussi se mettre à nu un petit peu, parce que la danse, c'est produire quelque chose personnellement, créer personnellement, [...], c'est un peu se mettre à nu devant tout le monde. » (H2) Ce qui est fondamental en danse est donc de « se détacher de la peur d'être vu et jugé » (H2). Les arguments formulés évoquent la thématique de l'individualisation, de la diversité, finalement difficile à gérer pour l'enseignant.

- Pour ceux qui ont inclus la danse, les propos évoquent : la motivation suscitée chez les élèves par la nouveauté et la découverte de la danse ou d'autres formes de danses, d'autres musiques; le fait qu'ils partent tous de niveaux très voisins ; la possibilité de 


\section{eJRIEPS 41 avril 2017}

respecter leurs différences; leurs progrès rapides et manifestes; l'acquisition d'une conscience corporelle différente de celle issue des autres activités physiques ; la facilité à obtenir un « résultat artistique » et propre à l'élève ; la possibilité que les élèves « peu sportifs » se sentent « revalorisés »; la moindre présence de l'affrontement compétitif et de la notion de performance au sens athlétique du terme ; la possibilité de raccrocher des élèves en échec et donc en refus face à la compétition permanente ; l'inclusion d'élèves parfois « introvertis » ou rejetés par leurs pairs ; la satisfaction des filles à rencontrer une activité que la majorité affectionne.

Ce sont donc les élèves, leurs progrès et découvertes qui sont évoqués par ces enseignants en réponse à la question des apports de la danse. Les acquis potentiels indépendants de la danse elle-même sont identiques à ceux du groupe précédent (échanger, respecter les avis des autres, gagner en autonomie...).

\section{3. 3 Danse ou cirque ?}

Quelles justifications pourraient conduire les enseignants à choisir entre danse ou cirque et accorder plus de place à l'une qu'à l'autre pratique en EPS ?

- Pour ceux qui enseignent seulement le cirque, le rapport technique/artistique y serait plus intéressant car : "Ça rassure aussi sûrement les collègues, parce qu'on peut se rapprocher à la technique. » (FC+) On peut solliciter « de l'acrosport, des manipulations d'objets, du jonglage, des quilles, des exercices sur boules. Je pense que c'est plus facilement manipulable pour les enseignants débutants. [...] T'as une fiche avec des niveaux de pratique sur les domaines acrobatiques, ou sur les manipulations, ça rassure énormément. » $(\mathrm{HC}+)$ L'effet « mode » de la présence du cirque en EPS est aussi évoqué. La diversité des objets et supports constitue un attrait certain pour les élèves : le zapping (entre équilibre, jonglage, acrobatie..) plaît ; les performances que représentent la maîtrise de l'équilibre en monocycle ou celle des coordinations en jonglage dopent leur motivation ; l'exécution d'acrobaties spectaculaires assure à ceux qui les réussissent un statut envié au sein du groupe de pairs; les garçons sont donc plus satisfaits et s'engagent facilement; les progrès sont plus rapides et manifestes qu'en danse. Aux yeux de ces enseignants, les collègues moins experts en cirque fuient la danse en survalorisant la dimension acrobatique du cirque aux dépends de sa dimension artistique.

- Pour ceux qui enseignent seulement la danse, ce rapport aux objets et à la technicité des manipulations est perçu de manière moins positive: des répétitions nombreuses sont indispensables avant d'accéder à un niveau satisfaisant qui procure du plaisir, ce qui peut 


\section{eJRIEPS 41 avril 2017}

décourager la plupart des élèves ; on peut se cacher derrière la technique et « oublier l'expression". Ce dernier argument laisse penser que, pour ces enseignants, la « technique » est plutôt entendue comme une suite de savoir-faire, répertoriés et classés, qui contribue à masquer, cacher quelque chose. II s'agit sans doute de la thématique de la créativité, dont l'étude montre qu'elle est conçue comme un strict produit de l'individualité du sujet (Cogérino, 2015).

- Pour ceux qui enseignent la danse et le cirque, les enjeux concernent la place relative de la dimension artistique, face aux entrées acrobatiques ou techniques en cirque, compte tenu de la spécificité du public des élèves et du temps nécessaire pour obtenir des progrès visibles: "La difficulté de jongler, de répéter de voir les balles tomber, de recommencer etc... et ça on a vraiment du mal, vraiment, vraiment...alors que la danse comme le mouvement part beaucoup plus naturellement d'eux-mêmes, euh... C'est quand même beaucoup plus facile d'avoir un résultat artistique à la fin [rire]. L'expression du corps et plus facile à atteindre, d'autant plus que le cirque, alors euh... y a cet aspect technique qui est super gênant parce que forcément même ceux qui rentrent dans la technique, finalement ils se cachent derrière ça et ils en oublient complètement l'expression. » (HCD+) Cette autre enseignante fait le choix inverse, elle « accentue aussi beaucoup sur le progrès technique, parce que le jeu d'acteur me paraît plus accessible dans d'autres activités aussi. Et donc en cirque, je suis plutôt sur une progression technique quand même pour que l'enfant soit valorisé : "voilà j'ai fait cirque, maintenant, je sais jongler." » (FCD+)

Pour HCD+, enseignant en ZEP/REP, «La danse, ça a un intérêt parce qu'on part du mouvement des élèves, y a pas besoin de savoir-faire techniques et on rentre directement dans une approche de l'artistique et d'expression libre ». Mais (FCD++), vraie spécialiste en danse et également en ZEP/REP, défend une approche plus singulière qui l'amène à positionner danse et cirque par rapport à l'abstraction gestuelle, et non par l'absence ou présence de la technique: « Je sais que les danseurs sont très contre le mime ; je sais que ce n'est pas toujours facile, parce que moi j'entre souvent par ça, pour les faire entrer dans la danse, avec du mime de la gestuelle quotidienne. Après on va transformer tout ça pour que cela devienne de la danse! Toute la partie abstraction, voilà, c'est ce qui la différencie du cirque !!! ». Elle incite aussi ses collègues à proposer ce type d'entrée pour le cirque : " Je leur ai dit la dernière fois: "Pourquoi vous ne feriez pas du cirque, par exemple toute la partie mime ?" Donc tu peux à partir du mime entrer par l'artistique pour 


\section{eJRIEPS 41 avril 2017}

sous-entendre la danse... Et eux m'ont dit "Ah non : si nous on fait du cirque, on sera plus sur le biais acro ! " Forcément, puisqu'ils ne sont pas dans un pôle artistique ! » (FCD++). Dans ces trois groupes d'enseignants, la question de l'accès à « l'expression » sans apprentissages préalables en danse affronte donc l'argument selon lequel cette même « expression » est difficilement accessible en cirque, mais aussi l'idée que « l'entrée acro » en cirque est plus séduisante pour les élèves. Ces arguments semblent bien le reflet de l'hexis corporel des enseignants d'EPS et de leur habitus sportif qui guide leur choix, argumentations et, au final, modalités d'enseignement (Garcia, 2013).

3. 4. Surprises et confirmations par l'analyse lexicale

À titre exploratoire, le repérage de 82 termes a été effectué afin de cerner une éventuelle différenciation thématique chez les enseignants en fonction de leur expérience d'enseignement dans l'une ou l'autre, ou aucune des deux pratiques, danse ou cirque (tableau 2).

Sur l'ensemble du corpus, les élèves sont mentionnés 295 fois. Mais la fréquence d'autres termes pour les évoquer est différenciée en fonction du sexe : danseurs et garçons sont plus nombreux que filles et danseuses et ce sont les spécialistes de danse qui utilisent davantage ces termes sexués. Ils mentionnent un peu plus que les autres les rôles sociaux de chorégraphe ou spectateur, tandis que les spécialistes de cirque utilisent plus que les autres les termes artiste et artistique.

Ceux qui n'enseignent ni cirque ni danse emploient plus fréquemment que les autres groupes les termes émotion, affect, sentiment, personnel, personnage, subjecti ${ }^{*} f$, " ve, ${ }^{*}$ vité), obstacle, réticen $\left({ }^{*} t\right.$, $\left.{ }^{*} c e\right)$, peur, appréhension et moins fréquemment le terme expression. Ceci renvoie au constat de l'intense préoccupation constatée chez ceux qui évitent la danse en raison de son lien avec la manifestation ou sollicitation d'émotions («trop personnel»), que celles-ci soient le starter de la créativité gestuelle ou la visée concernant les spectateurs. Ce même groupe est celui qui évoque le moins le regard.

Ceux qui enseignent surtout le cirque se distinguent par leur absence de mention de expression, person*, et leur faible abord de peur, émotion. Ce groupe est celui qui aborde le plus regard et technique. Le groupe moyennement compétent en danse se distingue par ses scores moyens à peu près partout sauf pour expression (score le plus fort) et subjectif (score le plus faible).

Le regroupement $\langle$ Sensation... $\rangle$ rassemble les termes ressenti, senti, sensation, proprioceptif, kinesthésique: il est davantage évoqué par les groupes pas ou peu 
compétents en danse. Les scores des termes proprioceptif, kinesthésique sont très faibles (3 mentions sur l'ensemble du corpus). II semblerait que les repérages sollicités concernent très peu la kinesthésie et sans doute davantage le ressenti du spectateur, au sens d'impressions suggérées par une gestuelle ou une prestation.

Les plus expérimentés en danse ont davantage mobilisé les termes du regroupement $\langle$ Modèle... > (montrer, démontrer, démonstration, modèle, imiter). Ce dernier constat est à mettre en relation avec une série de questions relatives aux procédures d'enseignement, partie de l'étude non rapportée dans cet article.

Compte tenu de la taille de l'échantillon, les différences numériques constatées sont à prendre avec distance. Elles confirment cependant un point déjà bien connu: les enseignants plus focalisés sur les garçons que sur les filles. Les données suggèrent aussi une focalisation de ceux qui n'enseignent ni cirque ni danse sur une dimension artistique organisée autour de l'émotion et des affects, ainsi que de la dimension « personnelle » à l'origine de la gestuelle ou de la thématique de la chorégraphie ou du numéro (ceci constituant un frein à leur engagement vers une pratique physique artistique).

Tableau 2. Vocabulaire spécifique en fonction de l'expérience d'enseignement en Danse.

\begin{tabular}{|c|c|c|c|c|c|c|}
\hline \multirow[t]{2}{*}{ Terme } & \multirow{2}{*}{$\begin{array}{c}\text { Nombre de } \\
\text { mentions } \\
\text { sur } \\
\text { l'ensemble } \\
\text { du corpus }\end{array}$} & \multicolumn{5}{|c|}{ Nombre moyen de mentions par enseignant } \\
\hline & & $\begin{array}{l}\text { Echantillon } \\
\text { total } \\
(n=14)\end{array}$ & $\begin{array}{l}\text { Enseignent } \\
\text { ni Danse ni } \\
\text { Cirque }\end{array}$ & $\begin{array}{l}\text { Spécialistes } \\
\text { Cirque }\end{array}$ & $\begin{array}{l}\text { Moyennement } \\
\text { compétents en } \\
\text { Danse }\end{array}$ & $\begin{array}{l}\text { Spécialistes } \\
\text { Danse }\end{array}$ \\
\hline Elève & 295 & 21.07 & 27 & 11.5 & 20.6 & 22 \\
\hline Gars/garçon & 50 & 3.57 & 0.33 & 1 & 2.6 & 8.75 \\
\hline Fille & 34 & 2.43 & 0 & 1 & 2.8 & 4.5 \\
\hline Danseur & 21 & 1.5 & 0.33 & 1 & 2 & 2 \\
\hline Danseuse & 5 & 0.36 & 0 & 1 & 0 & 0.75 \\
\hline Chorégraphe & 16 & 1.14 & 0 & 0.5 & 1.6 & 1.75 \\
\hline Spectateur & 17 & 1.21 & 2 & 0.5 & 2.4 & 1 \\
\hline $\operatorname{Artist}^{\star}(e$, ique $)$ & 50 & 3.57 & 2.33 & 6.5 & 3.8 & 2.75 \\
\hline Regard & 51 & 3.64 & 1.33 & 6.5 & 4.4 & 3 \\
\hline $\begin{array}{l}\text { Emotion }+ \text { affect }+ \\
\text { sentiment }\end{array}$ & 39 & 2.78 & 8 & 0.5 & 2.2 & 0.75 \\
\hline Personn ${ }^{\star}(\mathrm{el}$, age $)$ & 30 & 2.14 & 3,67 & 0 & 2,6 & 1,5 \\
\hline Subject $^{\star}$ (if, ivité) & 22 & 1.57 & 3 & 3.5 & 0.4 & 1 \\
\hline Expression & 18 & 1.29 & 0.67 & 0 & 2.6 & 0.75 \\
\hline $\begin{array}{l}\text { Obstacle }+ \\
\text { réticen* }(t, \text { ce })\end{array}$ & 16 & 1,14 & 2,67 & 1,5 & 0,6 & 0,5 \\
\hline $\begin{array}{l}\text { Peur+ } \\
\text { appréhension }\end{array}$ & 26 & 1.86 & 3 & 0.5 & 0.6 & 3.25 \\
\hline$\langle$ Sensations, ... & 44 & 3,14 & 4.67 & 5 & 4 & 2.5 \\
\hline$\langle$ Modèle, ... $\rangle$ & 147 & 10.50 & 7,33 & 8,5 & 9,2 & 15,5 \\
\hline Technique & 48 & 3,43 & 2,67 & 5 & 3 & 3,75 \\
\hline
\end{tabular}




\section{eJRIEPS 41 avril 2017}

\section{Discussion}

II s'agissait dans cette recherche de : 1. se demander si les FI des futurs enseignants d'EPS sont perçues suffisamment pertinentes dans leurs objectifs et procédures pour donner l'envie et la possibilité d'enseigner la danse à des étudiants majoritairement sportifs et peu connaisseurs des pratiques physiques artistiques ; 2 . clarifier le rapport établi par ces enseignants entre cirque et danse. Les recherches antérieures (cf. 1.2) ont souligné le rôle stratégique de la confiance des enseignants en leurs ressources pour enseigner la danse. L'étude des représentations des enseignants a donc questionné leur rapport au Savoir et au Pouvoir comme facteur de cette confiance, au sein d'entretiens restituant rétrospectivement leur FI vécue.

Les réactions des étudiants face à une même $\mathrm{FI}$ sont très variables. Certains découvrent un univers qui les enchante alors qu'il en fait fuir d'autres. Le sentiment de compétence de l'enseignant, construit sur l'évaluation du rapport entre Savoir et Pouvoir (Pujade-Renaud, 1976) est largement ébranlé chez ceux qui se déclarent non spécialistes de danse, insuffisamment formés. Concernant le Savoir, les repères habituels sont bousculés : ils se sentent peu sûrs de leurs objectifs et procédures d'enseignement, rapidement démunis quant aux choix de situations d'apprentissage qui répondent à leur représentation de la danse comme pratique artistique (l'expression, l'émotion, la créativité, la subjectivité ; pas ou peu d'apprentissages formels ou par reproduction-imitation...). Ils sont déstabilisés par le changement de repères et d'importance accordée à la motricité. Par ailleurs, ils se perçoivent en rupture par rapport à leur habitus professionnel : enseigner des pratiques sportives, codifiées et non «floues », facilement évaluables car les progrès des élèves y sont repérables, pouvoir à l'occasion montrer/démontrer aux élèves comment faire ou ne pas faire (Cogérino, 2015). De la sorte, la référence omniprésente à la « subjectivité » déclarée constitutive de la danse, l'incapacité ressentie à « démontrer » si nécessaire et à entrevoir des repères clairs pour situer les progrès des élèves aboutit à un renoncement à enseigner la danse en EPS. Dans le meilleur des cas, c'est le cirque, plus « sportivisable », mis en « fiches », déclaré plus « motivant » pour les élèves, qui assume la place d'une pratique artistique en EPS.

Notons que les entretiens recueillis en 1983-84 (Cogérino, 1988 ; 1999) font état de beaucoup de similarités avec ceux issus de la recherche présentée dans cet article, notamment pour les refus d'enseigner la danse et les arguments mentionnés pour compenser une $\mathrm{Fl}$ jugée insuffisamment professionnalisante. Le seul changement notoire 


\section{eJRIEPS 41 avril 2017}

tient au fait que les enseignants qui craignent ou refusent d'enseigner la danse peuvent actuellement y substituer le cirque. Mais si l'on prend en considération le nombre d'enseignants qui proposent la danse à leurs élèves, alors on pourrait avancer que, globalement, les $\mathrm{FI}$ actuelles rencontrent un échec relatif.

Trois thématiques émergent dans les entretiens face à la nécessité de donner confiance très tôt aux étudiants dans leurs capacités à animer une séance de danse avec des élèves : l'inefficacité ressentie des connaissances dites culturelles sur la danse ; la mise en défaut de l'habitus sportif des étudiants face à certains outils didactiques; la présentation contreproductive des courants de danse mettant en avant la « communication d'émotions » comme objectif premier de la danse.

Les enseignants ayant peu ou pas fréquenté les spectacles de danse se sentent en manque de repères sur son univers culturel, peu sûrs d'eux, dépourvus d'idées et perspectives pour son enseignement. Simultanément ils déclarent que ces connaissances «culturelles » ne leur semblent pas suffisantes pour savoir comment concevoir un cycle, gérer une séance, identifier les apprentissages accessibles, réguler leurs situations d'apprentissage. Pour une majorité d'interviewés, la danse est artistique parce que la prestation est un travail personnel, dépendant du ressenti, et donnant lieu à la création d'une gestuelle spécifique à chacun. La sollicitation de la créativité individuelle, la présence d'une émotion perceptible leur semblent des conditions sine qua non de la danse. La pratique scolaire proposée en EPS est dite artistique parce qu'on y est « vu » («on passe devant les autres », on est alternativement danseur, chorégraphe et spectateur) et la chorégraphie «transmet un message ». C'est ainsi une vision quelque peu démiurgique et très éloignée de la vie professionnelle réelle des danseurs et des circassiens, le plus souvent intermittents du spectacle, rudoyés en répétition et humiliés lors des auditions, davantage que chorégraphes encensés par la critique (Sizorn, 2008 ; Sorignet, 2004a, 2004b, 2006). Beaucoup d'aspects liés à l'individuation, l'unicité et singularité du sujet et sa production sont présents dans cette conception de l'artistique. Celle-ci tend à piéger les enseignants les plus « sportifs »: leur manque de confiance dans leur connaissance de la danse (le Savoir) est accentué par la culpabilité qu'ils ressentent à fixer des limites, donner un cap, proposer des procédures d'exploration des gestuelles ou thématiques, etc. (le Pouvoir): en bref, des procédures destinées à accroître la créativité des élèves. À leur décharge, il faut reconnaître que la brièveté des formulations des programmes pour les pratiques artistiques en EPS n'est guère aidante . 


\section{eJRIEPS 41 avril 2017}

Leur présenter le courant de la danse-théâtre, comme étant actuellement le plus abouti, c'est aussi accroître l'impression que danser c'est exprimer des émotions personnelles, ce qui génère réticences et rejets chez les plus éloignés de cet univers. La célébration du sensible en formation s'avère contreproductive et s'érige en obstacle au projet des formateurs/trices : accroître la pratique de la danse en EPS.

L'habitus sportif des étudiants s'avère mis en défaut lors de la présentation de la danse contemporaine par deux caractéristiques distinctes : la majoration de la centration sur l'émotion, celle du danseur tout comme celle des spectateurs, et l'absence de répertoires codifiés et facilement accessibles relatifs aux savoir-faire gestuels et techniques. Beaucoup se sont déclarés submergés, perdus face aux choix possibles de variables didactiques permettant de moduler les situations d'apprentissage.

Cela explique partiellement le succès du cirque qui peut être vu comme visant à « créer des effets » (effet visuel, comique, de surprise, de lenteur...) et pas seulement « créer des émotions », même si le spectateur en ressent. II semble donc échapper à l'image caricaturale de l'art aux dires des enseignants. Le cirque a ainsi l'image d'une pratique simultanément « sportive » car acrobatique, et artistique où les garçons ne craignent pas de s'investir. Ceci limite leur mise à l'écart volontaire, avec les moqueries et hors-tâches divers qui pourraient y être associés. L'accent porté lors de certaines formations sur un nouveau cirque esthétique et artistique, associé à la dévalorisation du cirque traditionnel, induit une mise à l'écart des filles, restreintes au rôle de faire-valoir des garçons et soumises à leur domination physique et symbolique (Garcia, 2007a, b ; Garcia \& Vigneron, 2006).

L'usage du terme «émotion » en formation suggère quelques remarques. Ce terme fonctionne comme un mot-valise, davantage synonyme de « ressenti de quelque chose» : cet usage est donc très éloigné de la liste des émotions primaires (joie, colère, dégoût, surprise, peur, tristesse) ou socialement élaborées (culpabilité, honte, ressentiment, admiration, etc.). L'emploi lors des FI et FPC des termes « effets » ou «impressions » à créer atténuerait les réticences générées, chez les étudiants et enseignants peu formés en danse, par l'injonction qui leur est faite d'émotions à procurer au spectateur ou qu'il faut «trouver en soi pour les exprimer». En outre, le statut et la fonction donnés à l'émotion fluctuent largement selon les courants de danse (Fouilhoux, 2014). Des courants s'appuyant sur les jeux de formes, une dimension ludique, la surprise (i.e. Alwin Nikolaïs), la composition aléatoire (i.e. Merce Cunningham) ou le respect de règles formelles 


\section{eJRIEPS 41 avril 2017}

(principes de répétition chez A. T. de Keersmaeker, dans ses premières chorégraphies, telle Rozas tantz rosas) éviteraient ces rejets a priori, relatifs à l'expression des émotions. II s'agit ici des conséquences des choix esthétiques effectués au sein de la culture chorégraphique pour amorcer la culture artistique en danse des étudiants, en limitant leur rejet initial face à son enseignement.

Les affres vécues par certains lors de leur FI en danse conduisent les moins proches de celle-ci à un oubli sélectif : celui-ci concerne les informations didactiques et pédagogiques inclus dans la $\mathrm{Fl}$. Cette partie de la formation est effacée des mémoires, refoulée, considérée comme absente ou très insuffisante. Dans les entretiens rétrospectifs sur la $\mathrm{FI}$, les interviewés déclarent qu'on leur a présenté les variables classiques permettant de moduler une gestuelle ou prestation dansée (en jouant sur le temps, l'espace, l'énergie, conformément au modèle de Laban). Mais ils se disent démunis dans l'usage qu'il faut en faire car submergés par les possibles évolutions générées par cette liste de variables. De la même façon que « connaître toutes les notes de la gamme », ou toutes les couleurs, ne permet pas nécessairement de construire une mélodie ou un tableau. À l'opposé, le discours tenu attribue à la FPC une qualité essentielle : celle de présenter des situations d'apprentissage aisément transposables dans les classes car "vécues » durant les stages de FPC. « Voir » comment un enseignant procède pour enseigner la danse permet aux plus anxieux ou réticents de banaliser leur inquiétude, d'oser animer une séance de danse. En FI, « faire connaître la danse » gagnerait peut-être à se moduler en « donner confiance à enseigner la danse ». Ceci engagerait un changement d'organisation important des $\mathrm{Fl}$ : par exemple animer quelques séances de danse en présence d'un tuteur, maître d'accueil, sur le modèle des stages temporaires. Les recherches consacrées à l'étude d'impact de cette procédure, présentées en Introduction, établissent que c'est un moyen sûr pour donner confiance aux étudiants en formation. Elle est par ailleurs courante dans la formation des enseignants du premier degré.

En conclusion, les $\mathrm{FI}$ impactent de manière conséquente l'étudiant qui découvre la pratique de la danse. Des réticences qui peuvent conduire au refus d'enseigner la danse y trouvent leurs racines. Et ce d'autant que l'habitus sportif des étudiants est déjà bien ancré pour la majorité d'entre eux. Les enseignants qui optent pour enseigner seulement le cirque considèrent que danse et cirque sont interchangeables, mais aussi que le cirque se rapproche intuitivement des pratiques «sportives»: la performance motrice s'y manifesterait sur des registres similaires (coordinations, rapidité, habileté manuelle, 


\section{eJRIEPS 41 avril 2017}

amplitude, prouesse, audace, etc.). Alors qu'en danse les modulations toniques, la valorisation du ralenti ou de mouvements de faible amplitude heurtent les repères habituels des enseignants. Peu d'enseignants de notre panel ont évoqué la nécessité de proposer aux élèves ces deux activités différentes, faute de temps d'enseignement peutêtre. Les raisons profondes, et sans doute davantage opérantes, résident plutôt dans l'articulation Savoir/Pouvoir construit et vécu par chaque enseignant. II reviendrait donc à la $\mathrm{FI}$ de reconsidérer ses procédures au service d'un changement d'objectif : donner d'abord confiance à l'étudiant dans ses capacités à animer des séances de danse, plutôt que de valoriser en premier "faire connaître la pratique culturelle de la danse ». Ce changement d'orientation des $\mathrm{FI}$, mis en application dans de nombreux pays, engagerait des procédures de formation telles que l'enseignement avec des petits groupes d'élèves, associé à un tutorat, sur des durées brèves mais qui permettent de mettre à distance le choc émotionnel vécu par une majorité d'étudiants sportifs lors de leur premier contact avec la danse contemporaine.

\section{Bibliographie}

Benhaïm-Grosse, P. (2007). Image du sport scolaire et pratiques d'enseignement au collège et au lycée. Les Dossiers, 190. Paris : Ministère de l'Education nationale, Direction de l'évaluation, de la prospective et de la performance.

Bourdieu, P. (1980). Le sens pratique. Paris : Editions de Minuit.

Cogérino, G. (1988). Les enseignants d'EPS face aux Activités Physiques d'Expression : quelle formation? Revue EP.S, 213, 28.

Cogérino, G. (1999). Les enseignants d'EPS face à l'enseignement de la danse. In CREEC (Ed.) La danse une culture en mouvement (pp. 197-205). Strasbourg : Editions université sciences humaines Marc Bloch.

Cogérino, G. (2015). Danse en EPS : les dilemmes du guidage vers la création. Spirale Revue de recherches en Éducation, 56, 81-96.

Connell, J. (2009). Dance education: an examination of practitioners' perceptions in secondary schools and the necessity for teachers skilled in the pedagogy and content in dance. Research in dance education, 10, 115-130.

Faure, S. \& Garcia, M.-C. (2003). Les «braconnages » de la danse Hip Hop dans les collèges des quartiers populaires. Vie-Ecole-Intégration Enjeux, 133, 244-247. 


\section{eJRIEPS 41 avril 2017}

Fouilhoux, B. (2014). Emotions et pratiques chorégraphiques. In G. Cogérino \& M.-C. Garcia (Eds.) L'EPS face au sensible et à l'artistique (pp. 313-324). Clapiers : AFRAPS.

Garcia, M.-C. (2007a). Le cirque en EPS. Rapport de recherche. Lyon, GRS, Centre Louise Labé, université Lyon2.

Garcia, M.-C. (2007b). Représentations «genrées » et sexuation des pratiques circassiennes en milieu scolaire. Sociétés et Représentations, 24, 129-143.

Garcia, M.-C. (2013). Le goût du cirque chez les enseignants d'EPS. STAPS, 102, 47-60.

Garcia, M.-C. \& Vigneron, C. (2006). Le cirque à l'école des banlieues. Agora Débats Jeunesse, 41, 32-48.

Green, L., Chedzoi, S., Harris, W., Mitchell, R., Naugthon, C., Rolfe, L. \& Stanton, W. (1998). A study of student teachers' perceptions of teaching the arts in primary schools. British educational research journal. 24(1), 95-107.

Hennessy, S., Rolfe, L. \& Chedzoy, S. (2001). The factors which influence student teachers' confidence to teach the arts in the primary classroom. Research in dance education, 2, 53-71.

Jodelet, D. (1984). Représentation sociale : phénomènes, concept et théorie. In $S$. Moscovici (Ed.) Psychologie sociale (pp.357-378). Paris : PUF.

Lesne, M. (1977). Travail pédagogique et formation d'adultes. Paris : PUF.

Lundvall, S. \& Maivorsdottir, N. (2010). Aesthetic aspects in meaning making - an explorative study of dance education in a PETE programme. Designs for learning, 3(1-2), 30-40.

Mac Lean, J. (2007). A longitudinal study to ascertain the factors that impacts on the confidence of undergraduate physical education student teachers to teach dance in Scottish schools. European physical education review, 13, 99-116.

McCormack, A.C. (2001). Using reflective practice in teaching dance to preservice physical education teachers. European Journal of Physical Education, 6, 5-15.

MENESR, (2008). Programmes du collège. Programmes de l'enseignement de l'éducation physique et sportive, Bulletin Officiel spécial ${ }^{\circ} 6$.

Necker, S. (2010). Faut-il danser pour faire danser à l'école ? Le corps de l'enseignant à l'épreuve de la transmission. STAPS, 89, 75-84.

Pujade-Renaud, C. (1976). Danse et narcissisme en éducation. Paris : ESF. 


\section{eJRIEPS 41 avril 2017}

Rolfe, L. (2001). The factors which influence primary student teachers' confidence to teach dance. European physical education review, 7, 157-175.

Sizorn, M. (2008). Une ethnologue en «trapézie » ; sport, art ou spectacle ? Ethnologie française, 38(1), 79-88.

Sorignet, P.-E. (2004a). Un processus de recrutement sur un marché du travail artistique : le cas de l'audition en danse contemporaine. Genèses, 57(4), 64-88.

Sorignet, P.-E. (2004b). Sortir d'un métier de vocation. Sociétés contemporaines, 56, 111132.

Sorignet, P.-E. (2006). Danser au-delà de la douleur. Actes de la recherche en sciences sociales, $163,46-59$.

Terret, T., Cogérino G. \& Rogowski I. (2006). Pratiques et représentations de la mixité en EPS. Paris : Editions Revue EP\&S. 


\section{eJRIEPS 41 avril 2017}

\section{Annexe}

Annexe 1. Grille simplifiée d'analyse de contenu.

La réalisation des entretiens, leur transcription et découpage ont été réalisés par Aurélie Gossoin, Cyril Devillers \& Thibault Loyer-Rohaut

\begin{tabular}{|c|c|c|}
\hline Macro-catégories & Subdivisions de niveau 1 & Subdivisions de niveau 2 \\
\hline $\begin{array}{l}\text { Itinéraire et vécu } \\
\text { personnel en Danse / } \\
\text { Cirque }\end{array}$ & $\begin{array}{l}\text { Modalités (types de pratique, volume } \\
\text { horaire, circonstances) } \\
\text { Apports perçus } \\
\text { Lacunes perçues }\end{array}$ & $\begin{array}{l}\text { Enfant/adolescent } \\
\text { Loisir adulte } \\
\text { Etudiant STAPS } \\
\text { Actuellement } \\
\end{array}$ \\
\hline $\begin{array}{l}\text { Descriptif de la formation } \\
\text { STAPS en Danse / Cirque }\end{array}$ & $\begin{array}{l}\text { Mentions de tâches, situations } \\
\text { d'apprentissage, organisation }\end{array}$ & $\begin{array}{l}\text { Descriptifs } \\
\text { Souvenirs positifs } \\
\text { Souvenirs négatifs }\end{array}$ \\
\hline $\begin{array}{l}\text { Conceptions de la } \\
\text { formation }\end{array}$ & $\begin{array}{l}\text { Avis sur la formation suivie / souhaitée } \\
\text { Réflexions/explications }\end{array}$ & $\begin{array}{l}\text { Positifs / négatifs } \\
\text { Propositions }\end{array}$ \\
\hline $\begin{array}{l}\text { Formation professionnelle } \\
\text { continuée }\end{array}$ & $\begin{array}{l}\text { Types d'apports perçus } \\
\text { Eléments appréciés } \\
\text { Descriptifs (tâches, séquences...) }\end{array}$ & $\begin{array}{l}\text { Professionnel } \\
\text { Relationnel } \\
\text { Personnel }\end{array}$ \\
\hline $\begin{array}{l}\text { Représentation de la } \\
\text { danse et évolution de } \\
\text { celle-ci }\end{array}$ & $\begin{array}{l}\text { Caractéristiques perçues de la danse } \\
\text { Apports/intérêts perçus pour les élèves } \\
\text { Evolution de la représentation chez } \\
\text { l'enseignant }\end{array}$ & $\begin{array}{l}\text { Pratique artistique / } \\
\text { physique / de santé } \\
\text { Evolution positive / } \\
\text { négative }\end{array}$ \\
\hline $\begin{array}{l}\text { Compétence perçue de } \\
\text { l'enseignant à enseigner }\end{array}$ & $\begin{array}{l}\text { En Danse } \\
\text { En Cirque }\end{array}$ & \\
\hline $\begin{array}{l}\text { Déclencheurs de } \\
\text { l'enseignement Danse / } \\
\text { Cirque en EPS }\end{array}$ & $\begin{array}{l}\text { Contraintes institutionnelles } \\
\text { Vécu et envie personnelle } \\
\text { Caractéristiques de l'établissement / des } \\
\text { élèves } \\
\text { Evènements particuliers }\end{array}$ & \\
\hline $\begin{array}{l}\text { Programmation de la } \\
\text { danse }\end{array}$ & $\begin{array}{l}\text { Eléments positifs } \\
\text { Eléments négatifs }\end{array}$ & $\begin{array}{l}\text { Pour les enseignants } \\
\text { Pour les élèves }\end{array}$ \\
\hline $\begin{array}{l}\text { Enseignement de la } \\
\text { danse }\end{array}$ & Difficultés perçues & $\begin{array}{l}\text { Chez les enseignants } \\
\text { Chez les élèves }\end{array}$ \\
\hline $\begin{array}{l}\text { Subjectivité perçue de la } \\
\text { danse }\end{array}$ & $\begin{array}{l}\text { De la danse « elle-même » } \\
\text { De son évaluation } \\
\text { De son enseignement }\end{array}$ & \\
\hline Pourquoi le cirque? & $\begin{array}{l}\text { Raisons de sa programmation / non } \\
\text { programmation } \\
\text { Adéquation aux caractéristiques des élèves }\end{array}$ & $\begin{array}{l}\text { Accélérateurs } \\
\text { Obstacles }\end{array}$ \\
\hline \multirow[t]{3}{*}{ Danse/cirque en ZEP } & $\begin{array}{l}\text { Caractéristiques perçues des élèves en } \\
\text { ZEP }\end{array}$ & En danse / en cirque \\
\hline & $\begin{array}{l}\text { Raisons de la programmation / non } \\
\text { programmation en ZEP }\end{array}$ & En danse / en cirque \\
\hline & $\begin{array}{l}\text { Intérêts perçus de l'enseignement } \\
\text { Danse/Cirque en ZEP }\end{array}$ & $\begin{array}{l}\text { En danse / en cirque } \\
\text { Selon que Danse et/ou } \\
\text { cirque sont programmés / } \\
\text { non programmés }\end{array}$ \\
\hline
\end{tabular}

NB : Il s'agit d'une grille simplifiée qui ne mentionne pas certaines thématiques développées dans d'autres publications en cours ; certaines sous-rubriques mentionnées ici ne sont pas exploitées dans cet article. 